\title{
Reproducción en cámara climática de las formas de alteración más frecuentes detectadas en materiales graníticos, en clima de tendencia continental
}

\author{
Fecha de recepción: 3-XI-98
}

Fecha de aceptación: 12-II-99

\author{
A.C.ÍNIGO,M.A.VICENTE y V.RIVES \\ IRNA-CSIC (Salamanca)
}

ESPAÑ A

\section{RESUMEN}

En el presente trabajo se intenta reproducir, mediante ensayos de envejecimiento acelerado en condiciones controladas, que reproducen los ambientes más frecuentes en zonas afectadas de clima de tendencia continental, las formas de deterioro más comunes que aparecen en edificios de interés historico-artístico, construidos con rocas graníticas en dichas zonas. Se analizan los cambios en la naturaleza y propiedades de las rocas (intensidad y rapidez), que se producen en las distintas experiencias.

En el estudio, realizado sobre granitos "sensu lato", se ha llegado a la conclusión de que el deterioro debido a procesos de frio/calor y hielo/deshielo es bastante lento $y$ poco apreciable a corto plazo, mientras que si interviene el fenómeno de cristalización de sales en las rocas, el deterioro detectado es rápido e intenso.

\section{SUMMARY}

This present work tries to reproduce, through test of controlated conditions in accelerate aging, reproducing the most frecuent ambients in places affected by a clima of continental tendency, the more commun forms of deterioration in historical interesting buildings, constructed with granitic rocks in those places.

We analyze the changes in the rocks nature and properties (intensity and rapidity), produced in different experiences.

In this study, realised on "sensu lato" granites, we come to the conclusion that the deterioration due to cold/hot and frost/defrosting processes is quite slow and not much appreciable within a short time, whereas if the phenomena of salts cristalization in the rocs occurs, the deterioration is rapid and intense.

\section{INTRODUCCIÓN}

El conocimiento de las características intrínsecas y la evolución de los materiales pétreos ornamentales, según el ambiente en el que han sido o van a ser empleados, es imprescindible tanto para un tratamiento racional del amplísimo Patrimonio Histórico construído con piedra, como para asegurar la calidad de las nuevas obras que constituirán el Patrimonio del Futuro [1].

La forma en que una piedra va a evolucionar frente a los factores extrínsecos al material (agua, contaminación, cambios bruscos de temperatura, etc.) depende de sus características intrinsecas y de las condiciones ambien- tales en que se sitúa. Es necesario, pues, conocer la respuesta del material al medio externo para prever su evolución. La naturaleza química y mineralógica, homogeneidad, textura, estructura, etc., de una piedra condicionan su comportamiento [1,2]. En rocas heterogéneas como el granito, formadas por distintas especies mineralógicas, algunas muy poco solubles, las formas de alteración más frecuentes son desplacaciones y arenizaciones; la evolución y/o disolución preferencial de alguna de las especies presentes puede dar lugar al desgranamiento del material [1].

La zona de trabajo escogida ha sido Ávila, ciudad declarada Patrimonio de la Humanidad por la UNESCO. Como 
edificio piloto para los estudios de observación y experimentación se seleccionó su Catedral.

Ávila está situada en el centro de la meseta castellana sobre una plataforma granítica, y bajo clima mediterraneo de tendencia continental. Los parámetros ambientales medios de la zona son [3]: Altitud, 1.100-1.200 m; temperatura media anual, $10^{\circ} \mathrm{C}$, con máximos y mínimos absolutos de $+39^{\circ} \mathrm{C} \mathrm{y}-20^{\circ} \mathrm{C}$ respectivamente, y precipitación anual de $400 \mathrm{~mm}$ (valores medios de los últimos 10 años).

El objetivo principal del trabajo es determinar la incidencia de los distintos factores de alteración en el deterioro obsevado en las piedras del monumento. Para ello se programaron una serie de ensayos de envejecimiento artificial (evolución acelerada) de las piedras, en condiciones controladas, en cámara de ensayos. En su diseño se intentó reproducir las condiciones ambientales más frecuentes en la zona de estudio.

\section{MATERIALES Y MÉTODOS}

En la Catedral de Ávila se observan diferentes tipos de deterioro sobre las cinco variedades de granito, que se han empleado para su construcción [1,4-6]. Los granitos estudiados van desde las facies naturales sanas [granito gris de grano fino (M-1) y granito gris de grano grueso (M-2)], con un bajo grado de porosidad y alta resistencia al deterioro, a facies naturales alteradas, con alta porosidad y especies fuertemente reactivas (arcillas expandibles, ópalo, etc.) ten su composición [granito ocre (M-3), granito rojo (M-4) y granito blanco (M-5)]. Su origen, propiedades mineralógicas, químicas y petrofísicas aparecen en trabajos anteriores [7-9].

Los bajos valores de contaminación atmosférica que se registran en Ávila hace que su efecto sobre el granito (rocas silicatadas, poco solubles y poco sensibles a los cambios de $\mathrm{pH}$ de las soluciones) sea casi inapreciable. El deterioro debido a deposición de contaminantes es sólo anecdótico. De aquí, que no se hayan realizado ensayos de deterioro por atmósferas contaminadas.

Las fuertes oscilaciones térmicas en la zona de estudio (Avila) parecen apuntar a un gran deterioro de la piedra debido a problemas de fatiga térmica y de heladas. Sin embargo, las zonas más deterioradas son aquéllas en que existe cristalización de sales. Numerosas citas bibliograficas corraboran este punto [5,6,10-12]. Con estas premisas se programaron las experiencias de envejecimiento artificial, sometiendo los diferentes materiales estudiados a bruscas variaciones térmicas y cristalización de sales. Así, en una cámara de simulación, marca ACS modelo UY600, con un microprocesador $\mathrm{J} 6000$, se sometieron probetas de las cinco variedades graníticas a ciclos de envejecimiento artificial, siguiendo las normas al uso. El control del deterioro producido en cada caso, se determinó por observación directa de las probetas y las pérdidas de peso sufridas por las mismas durante el ensayo. Los ensayos realizados fueron los siguientes:

\section{a) Frío/calor en seco $(-20 \text { a } 80)^{\circ} \mathrm{C}$}

Se realizó según el protocolo: probetas, previamente secadas hasta pesada constante, se colocan en la cámara climática 5 horas a $-20^{\circ} \mathrm{C}$. Posteriormente y con una rampa de calentamiento $50^{\circ} \mathrm{C} /$ hora, se eleva la temperatura hasta $80^{\circ} \mathrm{C}$ y se mantiene 4 horas a dicha temperatura [13].

\section{b) Hielo/deshielo (-20 a 110) ${ }^{\circ} \mathrm{C}$}

Se realizo segun la norma [14].

\section{c) Cristalización de sales $\left(\mathrm{SO}_{4} \mathrm{Na}_{2} \quad 10 \mathrm{H}_{2} \mathrm{O}\right)$}

Para programar este ensayo se tuvo en cuenta la naturaleza de las sales más frecuentemente encontradas en las distintas zonas del edificio, así como la contaminación del agua (pozos y aljibes) en la zona de la catedral. Se hizo de acuerdo al protocolo [15].

\section{d) Ensayo combinado}

En los edificios no se suelen dar los distintos procesos de degradación por separado, sino simultáneamente, con mayor o menor influencia de cada proceso, según los casos.

Por ello se programó un ensayo de envejecimiento artificial combinando cristalización de sales y hielo/deshielo [11]. El procedimiento seguido, una modificación del de Tiano y Pecchioni [14], es idéntico al seguido en el apartado de hielo/deshielo, pero en vez de en agua destilada, las probetas se sumergen en una disolución al 14\% de sulfato sódico decahidrato.

La modificación consiste en una suavización de las diferencias de temperatura empleadas $\left(-20^{\circ} \mathrm{C}\right.$ a $110^{\circ} \mathrm{C}$ en lugar de $-28^{\circ} \mathrm{C} \mathrm{a} 160^{\circ} \mathrm{C}$ ) y un aumento de la concentración salina ( $14 \%$ en peso en lugar de $10 \%)$ [13].

Al final del ensayo se lavan las probetas sumergiéndolas en agua destilada durante 48 horas, renovando el agua al menos dos veces. A continuación se procede a la última pesada, previo secado de las mismas a $60^{\circ} \mathrm{C}$ hasta peso constante.

\section{RESULTADOS}

Una vez que se hubieron realizado todos los ciclos de envejecimiento sobre cada una de las muestras estudia- 
das, se obtuvieron los siguientes resultados, según el tratamiento aplicado:

\section{a) Frío/calor}

En las probetas sometidas a 65 ciclos de envejecimiento acelerado (frio/calor, $-20^{\circ} \mathrm{C}$ a $80^{\circ} \mathrm{C}$ ), el grado de deterioro provocado por este ensayo es casi imperceptible. No se detectaron pérdidas de peso apreciables y el aspecto exterior de las mismas apenas varía.

\section{b) Hielo/deshielo}

Veinticinco ciclos de hielo/deshielo $\left(-20\right.$ a $\left.110^{\circ} \mathrm{C}\right)$, provocaron en las probetas los siguientes efectos:

-En los granitos Grises (M-1 y M-2) no se detectaron apreciables pérdidas de peso. Los únicos cambios observables son la oxidación de las biotitas superficiales y el desprendimiento superficial de alguna laminilla de mica.

-En las variedades M-3, M-4 y M-5 se detectó una ligera disminución de peso de las probetas durante el envejecimiento $(<0,3 \%)$, que, probablemente, es debida a un arrastre de arcillas de los poros (se trata de variedades con esmectita, caolinita, ópalo y oxihidróxidos de hierro), en los ciclos de inmersión y escurrido de las probetas y/o a ligeras disoluciones.

\section{c) Cristalizacion de sales}

En las probetas sometidas a ciclos de cristalización de sales con $\mathrm{SO}_{4} \mathrm{Na}_{2} \cdot 10 \mathrm{H}_{2} \mathrm{O}$, siguiendo la norma [13], se observaron los siguientes cambios:

-El granito Gris (M-1 y M-2) no presenta alteración aparente después de 20 ciclos, excepto oxidación de las biotitas superficiales.

-La variedad Ocre (M-3) presenta una fuerte degradación superficial tras tres ciclos de tratamiento (Foto 1).

-En la Piedra Sangrante (M-4 y M-5) la degradación superficial empieza a apreciarse a partir de los ocho ciclos y con menor intensidad.

\section{d) Ensayo combinado: cristalizacion de sales-hielo/ deshielo}

Tras los primeros ciclos de envejecimiento, siguiendo el ensayo combinado, no se apreció deterioro superficial en las probetas, como ocurría en el ensayo de cristalización de sales, pero entre los ciclos 15 y 18 aparecen grietas, que, en los 3 ciclos posteriores, producen fuertes resquebrajamientos, llegando a alcanzar una profundidad de 0,5 a $1,5 \mathrm{~cm}$, sobre todo en las muestras M-3, variedad que contiene esmectita en su composicion (Foto 1).

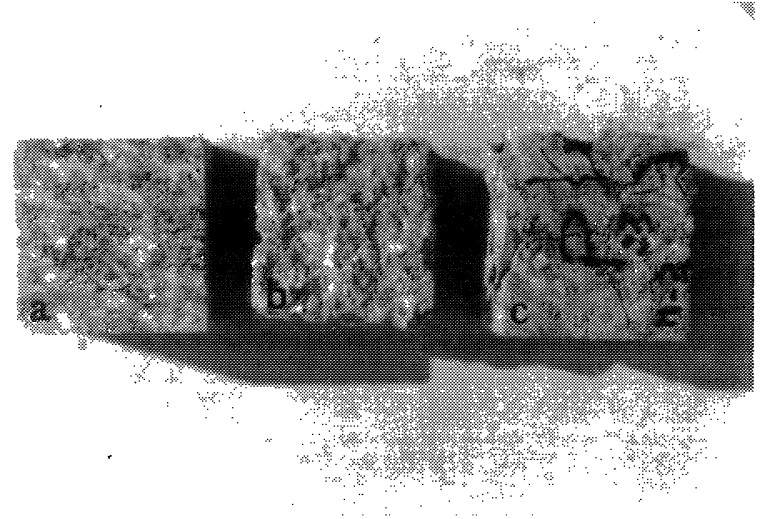

Foto 1.- Probetas envejecidas artificialmente: a) testigo, b) después de 10 ciclos de cristalización de sales [RILEM, 1980]: fuerte deterioro superficial, y c) después de 18 ciclos de cristalización de sales (ensayo combinado de cristalización de sales y hielo/deshielo):fracturación.

La diferencia de efectividad entre el "envejecimiento" por cristalizacion de sales y el combinado ( hielo/deshielo-cristalizacion de sales) puede deberse a lo siguiente:

En el primer caso, el agua accede a la superficie de secado con una gran carga iónica y provoca una fuerte y rápida cristalización de sales en la parte mas externa de la piedra, generando fuertes tensiones, que provocan su rapido deterioro (Foto 1).

En el ensayo combinado (sales, hielo/deshielo), previo al secado, tiene lugar una congelacion. Durante la misma, gran parte de los iones que lleva la disolución salina, precipitan en el interior de la piedra y, en el caso de las muestras M-3, M-4 y M-5, que tienen en su composición arcillas, quedan adsorbidas en éstas. En el posterior secado, la solucion que llega a la superficie, está notablemente menos cargada en sales y el efecto disruptivo de las mismas en los primeros ciclos se amortigua. Sin embargo, al repetir el proceso ( 15 ciclos) la carga salina en el interior de la piedra es tan grande que provoca la rotura total de ésta, Foto 1.

Este efecto se observa en el edificio en zonas expuestas a fuertes cambios térmicos y con afluencia de soluciones salinas, debidas a disoluciones de morteros (Girola), Foto 2 .

Con los distintos ensayos se han reproducido buena parte de las patologías encontradas en la catedral de Avila. Así pués, por observación de las patologías presentes (efectos) y analizando las condiciones ambientales, se puede predecir las formas dominantes de degradación que sufrirán.

Los resultados obtenidos por Tiano y Pecchioni [14], Martín y col. [16] y Esbert y col. [17] sobre diferentes naturalezas de materiales pétreos de cantera están de 


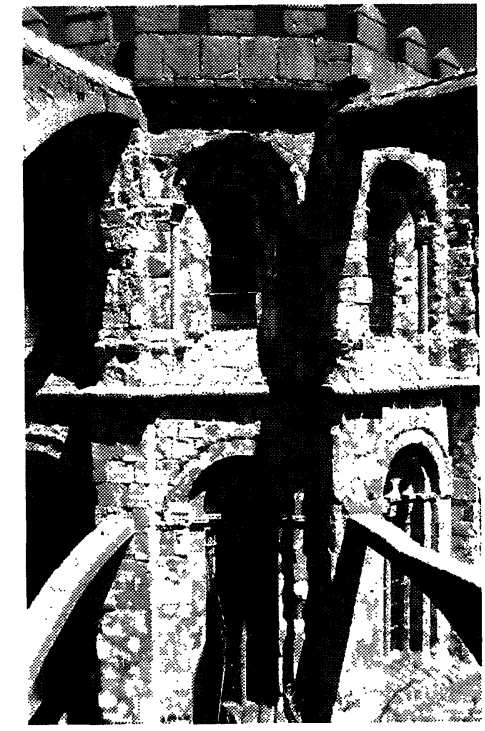

Foto 2.- Piedra sangrante degradada (Girola, catedral de Ávila).

acuerdo en cuanto al orden de deterioro alcanzado, por los resultados de los diferentes tipos de envejecimiento acelerado utilizados sobre nuestras muestras. La intensidad de deterioro es la siguiente:

Frio/calor $<$ Hielo/deshielo $<$ Cristalización de sales $<$ Combinado(sales + hielo/deshielo)

\section{CONCLUSIONES}

El estudio de envejecimiento acelerado sobre los distintos tipos de rocas graníticas estudiadas permite establecer que:

1) El proceso de envejecimiento que provoca un deterioro más rápido e intenso es la cristalización de sales.

2) El ensayo más agresivo resulta ser aquél en el que interviene la cristalización de sales junto con ciclos de hielo/deshielo, ya que provoca un deterioro más profundo.

3) Los ensayos que someten los materiales a ciclos de frío/calor y hielo/deshielo por separado resultan mucho menos agresivos, sobre todo el primero. Incluso despues de 65 ciclos, el deterioro observado en la piedra, es mínimo.

4) El evitar el acceso de humedad a las piedras monumentales, sea de remonte capilar, filtraciones o cualquier otra vía, es la forma más efectiva de prevenir su deterioro.

5) La acción sinérgica de distintos factores de alteración potencia notablemente el deterioro producido por cada uno de ellos.

\section{AGRADECIMIENTOS}

Los autores agradecen la ayuda de la CE (STEP CT90-
0101), Junta de Castilla y León (proyecto 0314/90 y becario) y CICYT (PAT91-1507-C03).

\section{BIBLIOGRAFÍA}

(1) Vicente, M. A.: 1994. "La piedra, un valioso recurso natural". Fronteras de la Ciencia y la Tecnología, 6, 50-54.

(2) Olmos, C.: 1991. "Patología de la piedra y del ladrillo. Curso de "Patología, conservación y restauración de edificios". Ed. Servicios de publicaciones del Colegio de Arquitectos de Madrid, n 190-191, 85-95.

(3) García-Talegón, J., Íñigo, A. C., Molina, E., Rives, V., Vicente, M. A., 1993. "Granitos empleados en la Catedral de Avila: características de los materiales de cantera". Proc. "Alteracion de granitos y rocas afines", 125-128.

(4) Rodríguez-Caro, C.: 1993. "La restauración de la Catedral de Avila". Proc. "Alteracion de granitos y rocas afines", 93-97.

(5) Arango-Diez, J.: 1993. "Proyecto de restauración del Claustro de la Catedral de Avila". Proc. "Alteracion de granitos y rocas afines", 98-102.

(6) Vicente, M. A.: 1995. "Summary of Project STEP-CT900101, Granitic materials and historical monuments: study of the factors and mechanisms of weathering and application to historical heritage conservation". Proc. Degradation and conservation of granitic rocks in monuments, Vicente M. A., Delgado-Rodrigues J., Acevedo, J. (Eds.), 4-44.

(7) García-Talegón, J. Molina, E., Vicente, M. A.: 1991. "Weathering processes in granites". Proceedings del $7^{\circ}$ Congreso Europeo de Arcillas-EUROCLAY, Dresden (Alemania), vol. 2,405-409.

(8) García-Talegón, J., Íñigo, A. C., Molina, E., Pérez-Rodríguez, J. L., Vargas, M., Vicente, M. A., 1994. "Granites employed in Avila (Spain): I.- Chemical composition of the differentss types". Materiales de Construcción, 44, 23-28.

(9) İñigo, A. C., García-Talegón, J., Molina, E., Pérez-Rodríguez, J. L., Vargas, M., Vicente, M. A.: 1994. "Granites employed in Avila(Spain): II.- Petrophysical characteristics". Materiales de Construcción, 44, 28-37.

(10) Arnold, A., Zehnder, K.: 1985. "Crystallization and habits of saltefflorescences on walls, part II: Condition of crystallization. $5^{\text {th }}$ Int. Cong. Deterior. Conserv. Stone, Lausanne, 269-277.

(11) Arnold, A., Zehnder, K.: 1989. "Salt weathering on monuments. In: F. Zezza (Editor), The Conservation of Monuments in the Mediterranean Basin. Grafo Edizione, Bari, 31-58.

(12) Evans, I. S.: 1970. "Salt crystallization and rock weathering:a review"Rev. Géomorphol. Dynam., 19, 153-177.

(13) Íñigo, A. C.: 1995. Tesis de Doctorado, Salamanca.

(14) Tiano, P.,Pecchioni, E.:1990. "Invecchiamento artificiale di materiali lapidei". Proceeding di giornata di studio "Camera climatiche od ambientali nella ricerca applicata", 37-42.

(15) RILEM: 1980. "Essais recommandeés pour l'altération de pierres", test V. la.

(16) Martín, L., Bello, M. A., Martín, A.: 1992. "Accelerated alteration tesis on the stones used in the cathedral of Granada (Spain)". Proc. " $7^{\text {th }}$ International Congress on Deterioration and Conservation of Stone", 845-850.

(17) Esbert, R. M., Marcos, R. M., Ordaz, J., Montoto, M., Alonso, F. J., Suárezdel Río, L. M., Ruizde Argandoña, V.G., Calleja, L., Rodríguez Rey, A.: 1991. "Petrografía, propiedades físicas y durabilidad de rocas utilizadas en el patrimonio monumental de Cataluña, España ( $2^{\circ}$ parte)", Materiales de Construcción, 41,49-59. 